\title{
Effet de l'activité des insectes pollinisateurs sur la pollinisation et le rendement du tournesol de consommation
}

\author{
André Fougeroux ${ }^{1, *}$, Solenne Leylavergne ${ }^{1}$, Vincent Guillemard ${ }^{2}$, Olivier Geist ${ }^{3}$, Pauline Gary ${ }^{4}$, \\ Charlotte Cenier ${ }^{4}$, Edith Caumes-Sudre ${ }^{5}$, Christopher Senechal ${ }^{1}$ et Bernard Vaissière ${ }^{6}$ \\ ${ }^{1}$ Syngenta, 12 Chemin de l'Hobit, 31790 Saint-Sauveur, France \\ 2 Master 2 Sciences du Végétal, Université Paris Sud, 91405 Orsay cedex, France \\ ${ }^{3}$ Master 2 Biologie et valorisation des plantes, Université de Strasbourg, 67000 Strasbourg, France \\ 4 Agrosolutions, 83 Avenue de la Grande-Armée, 75016 Paris, France \\ 5 Arterris Innovation, 24 Avenue Marcel Dassault, 31500 Toulouse, France \\ ${ }^{6}$ INRA, UR406 Abeilles et Environnement, 84914 Avignon cedex 9, France
}

Reçu le 29 août 2017 - Accepté le 31 octobre 2017

\begin{abstract}
Résumé - La pollinisation entomophile du tournesol est souvent mentionnée comme un facteur contribuant au rendement et à la qualité de cette culture. Alors que les rendements stagnent depuis une trentaine d'années en France, l'amélioration de la pollinisation des tournesols de consommation constitue une piste pour les relancer en conditions agricoles. Notre étude visait à évaluer l'impact d'un front d'insectes pollinisateurs (colonies d'abeilles domestiques installées dans une bande fleurie disposée sur une bordure de parcelles agricoles longue de plus de $550 \mathrm{~m}$ de long). Cet impact a été mesuré à différentes distances de ce front d'insectes pollinisateurs sur la fréquentation des capitules par les insectes floricoles, la charge en pollen des stigmates, le rendement et la teneur en huile des graines. Nous avons bien observé un gradient négatif d'activité des abeilles domestiques en fonction de l'éloignement au front de colonies et aussi une diminution du rendement et de la teneur en huile chez le tournesol. Nous présentons la méthodologie originale utilisée ainsi que les premiers résultats obtenus.
\end{abstract}

Mots clés : pollinisation / tournesol / abeille / rendement / teneur en huile / gradient

Abstract - Effect of the activity of pollinators insects on pollination and yield of commercial sunflower. Insects' pollination on sunflower is often presented as a key factor contributing to the yield and quality of this crop. Yields of sunflowers have been stagnant over the past 30 years in France, and improvement of sunflower pollination has been proposed as a mean to increase yields in agricultural settings. Our study aimed at evaluating the effect of a front of pollinators (honeybee colonies placed in flowering margins set along one side of sunflower fields over $550 \mathrm{~m}$ long). This effect was measured at different distances from this pollinator front on the forager density on sunflower heads, the stigmatic pollen loads, and the yield and oil content of the seeds. We did find decreasing honeybee densities on sunflowers at increasing distances from the colony front, and also decreasing sunflower yield and oil content. We present the original methodology used and our first results.

Keywords: pollination / sunflower / bee / yield / oil content / gradient

\section{Introduction}

La pollinisation entomophile (par les insectes) des cultures fait l'objet de nombreuses déclarations, souvent extrêmes. Effectivement, le rôle des insectes pollinisateurs est important pour la reproduction de nombreuses plantes à fleurs et la

\footnotetext{
$\overline{* \text { Auteur de correspondance }}$ : fougeroux.andre@outlook.com
}

contribution à l'agriculture a été chiffrée à environ 1,4 milliards d'euros en France (Leonhardt et al., 2013). Le service écosystémique des insectes pollinisateurs est donc indéniable, toutefois l'intérêt économique pour les agriculteurs reste à préciser.

La pollinisation entomophile en production de semences hybrides de tournesol a fait ses preuves, en termes de quantité de semences et de qualité des lots produits. Mais l'intérêt de la pollinisation par les abeilles en production de tournesol de 
consommation n'est pas clairement établi, et la plupart des agriculteurs ne sont pas convaincus de la valeur économique de ce service.

Pour satisfaire les demandes alimentaires et industrielles en besoins croissants en huile et matières grasses, on se heurte à la stagnation de la production et à une baisse des stocks mondiaux en graines oléagineuses. Une des raisons de ce phénomène est l'absence de croissance des rendements du tournesol. Celle-ci peut avoir plusieurs causes, comme le changement climatique ou le raccourcissement des rotations. Le progrès génétique n'est toutefois pas mis en cause. En effet, on estime que la sélection variétale du tournesol s'est traduite par un gain moyen de 0,5 quintaux/ha/an par rapport aux anciennes variétés (GNIS, 2016). En conditions de culture optimale, on estime que les variétés actuelles peuvent produire de 60 à 70 quintaux par hectare. En comparaison, la moyenne nationale reste autour de 23-24 quintaux/ha. De fait, on observe que depuis les années 1980, le rendement en tournesol stagne (ONIDOL, 2012). Comment expliquer cette stabilité des rendements depuis autant d'années? Et comment expliquer la différence de rendement avec ceux obtenus en conditions optimales de culture?

De fait, le tournesol est considéré comme une plante relativement résiliente, pouvant être cultivée en plein champs sans irrigation et avec très peu d'engrais. Cette idée empêcherait les variétés d'exprimer leur potentiel maximum.

Le taux d'autofertilité des variétés de tournesol est variable, de sorte qu'une meilleure pollinisation, notamment par un recours à l'activité pollinisatrice d'abeilles, a été proposée depuis longtemps comme un moyen de déplafonner les rendements (Taséi, 1984). En effet, la pollinisation est une étape clé dans la fécondation et la formation des graines. Elle correspond à la libération, au transport, puis au dépôt des grains de pollen sur le stigmate suivis de l'acheminement des noyaux spermatiques jusqu'aux ovules. Pour le tournesol, les grains de pollen peuvent être transférés des organes mâles vers les organes femelles selon trois modes : le vent, en véhiculant les grains de pollen et en agitant les plantes (pollinisation anémophile), la gravité ou le contact direct entre les anthères et les stigmates (autopollinisation passive pour les variétés autofertiles) et les insectes (pollinisation entomophile).

Comment atteindre la pollinisation optimale qui permette l'expression maximale du potentiel de la plante, compte tenu des ressources disponibles (eau, fertilisation, état sanitaire...)? Des études ont été conduites sur tournesol par l'INRA et Syngenta en 2015 et en partenariat étendu avec Agrosolutions et Arterris en 2016, afin d'évaluer en conditions pratiques l'intérêt agronomique de l'activité des insectes pollinisateurs. Ces travaux ont été menés dans la région toulousaine sur des parcelles d'agriculteurs. Préalablement à la mise en place des travaux expérimentaux, une succincte revue bibliographique a été réalisée.

\section{Revue bibliographique}

Sur tournesol, les abeilles domestiques sont les insectes floricoles les plus nombreux (Carvalheiro et al., 2011), et elles sont aussi considérées comme le principal agent pollinisateur de cette culture dans le monde entier (Krishna et al., 2014; Nderitu et al., 2008). Cependant, il semble que l'efficacité de la pollinisation entomophile soit conditionnée par la diversité morphologique et comportementale des abeilles sauvages et domestiques présentes, avec une possible complémentarité entre les espèces (Carvalheiro et al., 2011; Garibaldi et al., 2013). De nombreux auteurs ont démontré l'avantage du service écosystémique rendu par les abeilles en comparant les rendements de plantes sous tulle pour lesquelles la pollinisation ne s'effectue que par autopollinisation passive et par le vent avec ceux de plantes exposées aux insectes pollinisateurs en pollinisation libre. Une première étude a eu lieu sur la variété 1957 de Bulgarie en comparant 60 capitules en pollinisation libre à 60 capitules sous tulle. Le gain de rendement lié à la pollinisation entomophile était de $+220 \%$ et le gain d'huile de $+214 \%$ (Barbier et Abid, 1966). Avec une méthode similaire, Nderitu et al. (2008) obtient sur l'Hybrid 8998 un gain de rendement de $115 \%$ la première année et de $107 \%$ la seconde année, avec un gain d'huile mesuré cette deuxième année de $+167 \%$. Enfin, avec cette même méthode, Krishna et al. (2014) sur la variété SFL 8 obtiennent un gain de rendement de $23 \%$ en faveur de la pollinisation libre.

Comme on peut le constater, il y a de grandes disparités dans ces gains de rendement obtenus en comparant les capitules sous tulle et les capitules en pollinisation libre. Ces variations importantes peuvent provenir de différences entre les types de tulle utilisés dont les mailles peuvent laisser passer plus ou moins de pollen et surtout de différence dans le niveau d'autofertilité des variétés utilisées qui ne sont jamais indiquées. Mais ces résultats reposent la question de l'optimisation de la pollinisation pour maximiser le rendement et la teneur en huile des variétés actuelles de tournesol de consommation sous différentes conditions agroenvironnementales.

\section{Protocole et dispositif experimental}

Nous nous sommes inspirés d'un protocole de la FAO réalisé pour détecter les déficits de pollinisation (Vaissière et al., 2011).

Un total de cinq parcelles d'étude (parcelles d'agriculteurs) a été suivi en 2015 et six en 2016 dans la région située entre Toulouse et Castelnaudary. Sur chaque parcelle, un dispositif identique a été implanté. Les parcelles doivent mesurer environ $600 \mathrm{~m}$ de long, être le plus homogène possible, en évitant les parcelles en pente. Sur chaque parcelle, une bande fleurie est semée perpendiculairement à la plus grande longueur de la parcelle (Fig. 1). Le tournesol est implanté par l'agriculteur. En 2015, une seule variété a été utilisée: Extrasol. En 2016, les agriculteurs étaient libres du choix de leur variété. En juin avant la floraison du tournesol, des colonies d'abeilles domestiques sont disposées sur la bande fleurie et des zones de relevés (placettes) sont matérialisées à trois distances de ce front de colonies. Afin de relever un éventuel gradient d'insectes pollinisateurs, ces placettes sont espacées de 250 mètres à partir des colonies, soit à $30 \mathrm{~m}, 280 \mathrm{~m}$ et $530 \mathrm{~m}$ de celles-ci, respectivement. Trois placettes de relevés (traitées comme 3 répétitions) étaient installées à chaque distance (Fig. 1).

La bande de récolte correspond à la zone dans laquelle sont prélevés 30 capitules en face de chaque placette pour estimer le rendement agriculteur par distance. 


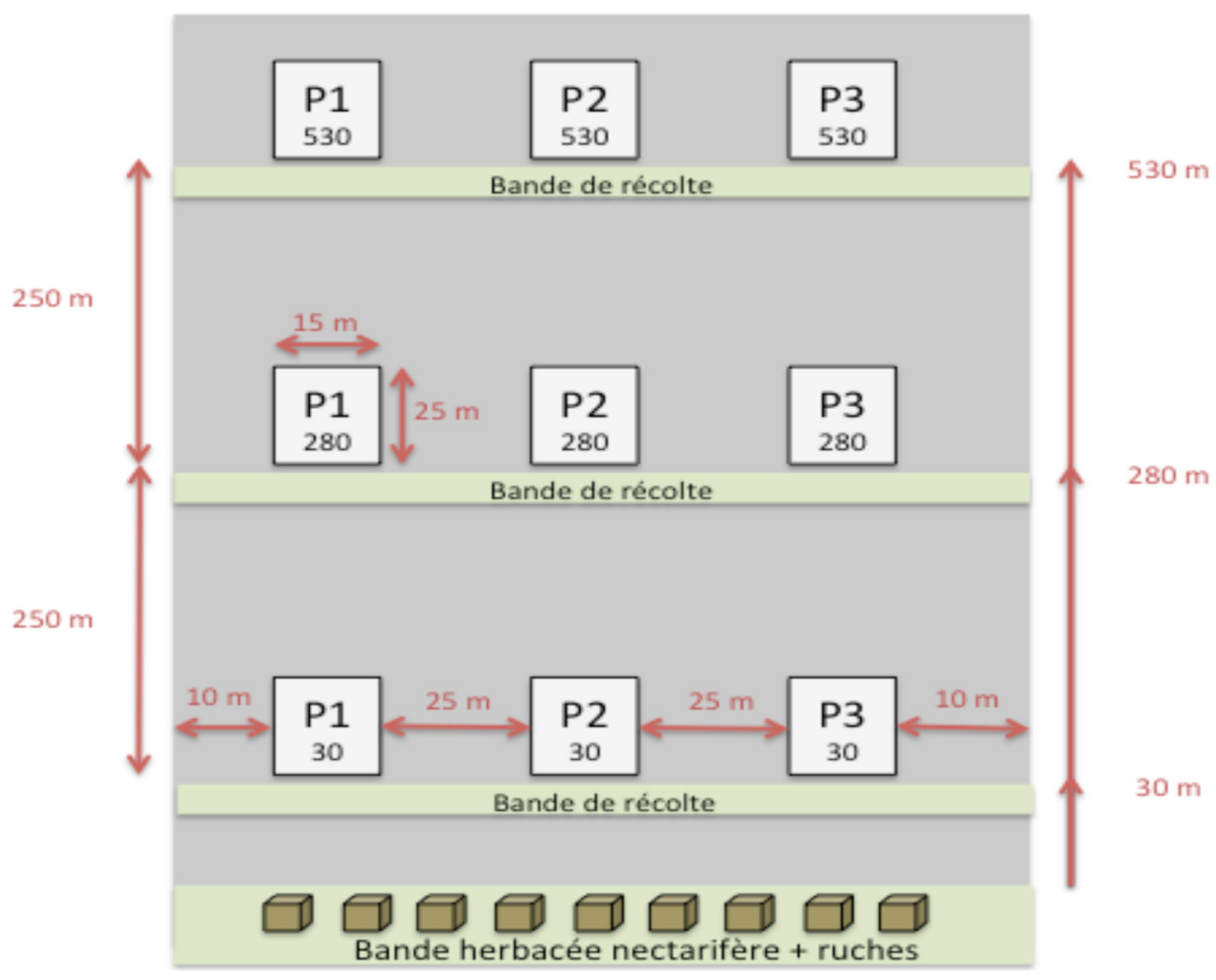

Fig. 1. Disposition des placettes de relevés sur chaque parcelle à trois distances du front de colonies d'abeilles domestiques installé sur la bande nectarifère avec le gradient attendu de densité d'insectes pollinisateurs et de niveau de pollinisation.

Fig. 1. Locations of the test plots on each field at three distances from the honeybee colonies set amidst the band of nectariferous plants with the expected gradient of pollinator density and pollination level.

Tableau 1. Composition du mélange I-Sol ${ }^{\circledR}$ PRONECTAR.

Table 1. Composition of the flowering mix I-Sol ${ }^{\circledR}$ PRONECTAR.

\begin{tabular}{llcl}
\hline & Composition (\% du poids) & Période de floraison & Cycle de vie et sensitivité au gel \\
\hline $\begin{array}{l}\text { Sainfoin } \\
\text { Onobrychis sativa }\end{array}$ & 55 & Mai-août & Pérenne \\
$\begin{array}{l}\text { Mélilot blanc } \\
\text { Melilotus alba } \\
\text { Trèfle violet } \\
\text { Trifolium pratense }\end{array}$ & 10 & Juin-juillet & Bisannuelle \\
$\begin{array}{l}\text { Trèfle de Perse } \\
\text { Trifolium resupinatum } \\
\text { Trèfle incarnat } \\
\text { Trifolium incarnatum }\end{array}$ & 10 & Mai-juin \\
Phacélie & 10 & Juin-juillet \\
Phacelia tanacaetifolia & 10 & Mai-juin & Pérenne \\
& 5 & 2 mois après semis & Annuelle et gélive \\
\end{tabular}

\subsection{Mise en place de la bande nectarifère}

Les bandes nectarifères ont été implantées en début de saison (fin mars en général) quelques jours avant le semis du tournesol. La surface de ces bandes représentait $3 \%$ de la surface de la parcelle de tournesol soit $300 \mathrm{~m}^{2}$ par hectare de tournesol. Après une préparation soignée du lit de semence, on semait le mélange I-Sol ${ }^{\circledR}$ PRONECTAR (Semences de France, la Chapelle d'Armentières, France) à $0,5 \mathrm{~cm}$ de profondeur et une dose de $40 \mathrm{~kg} / \mathrm{ha}$. Ce mélange pluriannuel avait pour objectif de fournir une zone de nidification et d'alimentation au printemps avant la floraison des tournesols pour les insectes pollinisateurs sauvages, de façon à augmenter leur population sur les parcelles d'étude (Tab. 1). 


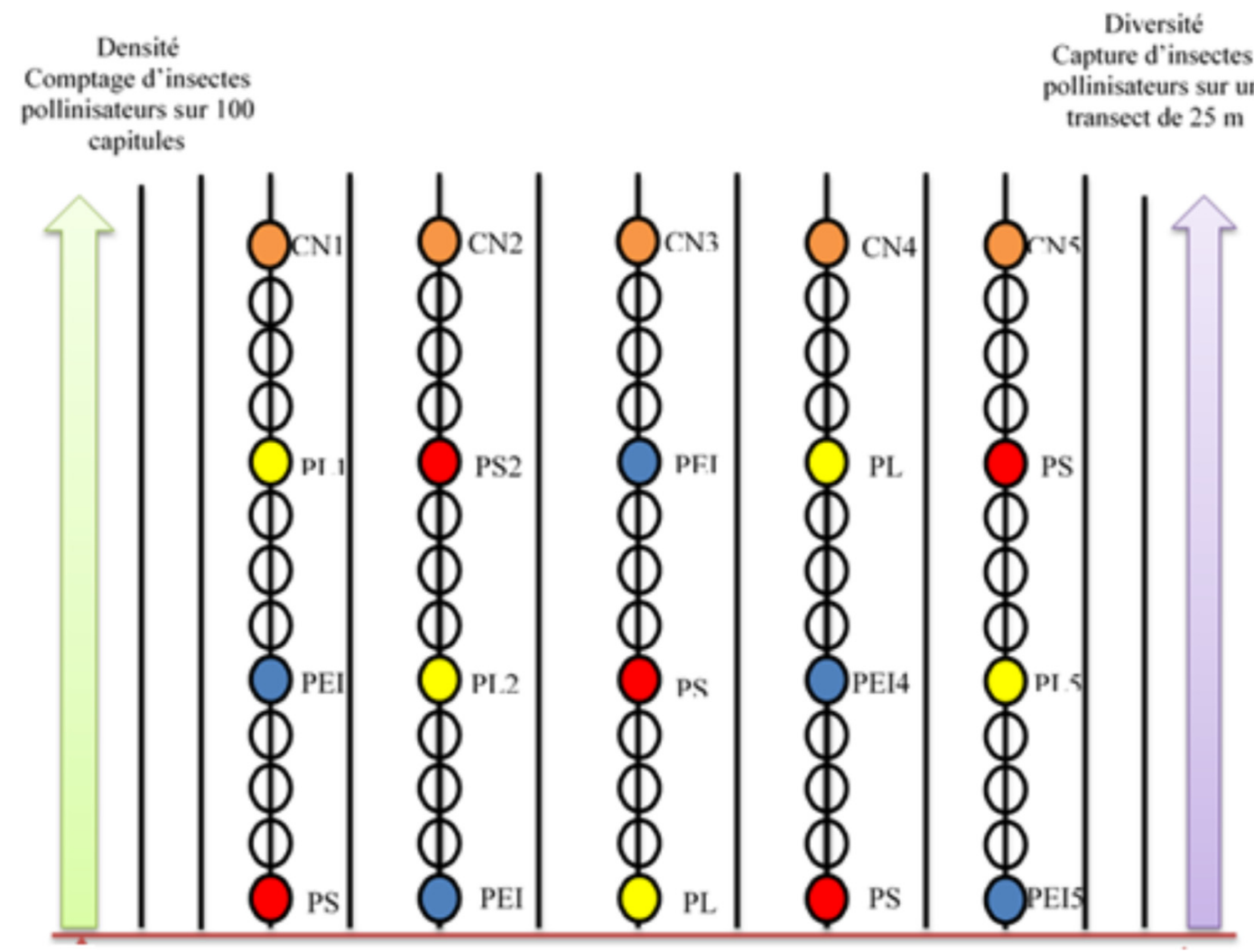

Fig. 2. Organisation d'une placette de relevés avec les mesures de densité et de diversité d'insectes pollinisateurs et les cinq capitules avec les trois traitements (pollinisation tulle PEI, pollinisation libre PL et pollinisation saturante PS) randomisés au sein de chaque rang. Les cinq capitules orange indiquent la localisation des capitules utilisés pour mesurer la production nectarifère (placette centrale de la parcelle uniquement).

Fig. 2. Set-up of a test plot with rows used to record the density and diversity of pollinators and the five capituli used for each of the three treatments randomized within each row (tulle PEI, open pollination PL and saturation hand pollination PS). The five orange heads indicate the location of the heads used to measure the nectar production (central plot in each field only).

\subsection{Installation des colonies d'abeilles domestiques}

Les colonies ont été installées sur les bandes nectarifères avant le début de leur floraison. Une charge nominale de deux colonies par hectare de tournesol a été visée, afin d'avoir un nombre suffisant de butineuses pour espérer observer un gradient de butinage sur $500 \mathrm{~m}$, sans pour autant saturer la zone en insectes pollinisateurs sauvages. Toutes les colonies étaient issues du même apiculteur afin d'éviter autant que faire se peut des biais liés à des pratiques apicoles différentes.

\subsection{Mesure de l'activité des pollinisateurs}

La densité d'insectes pollinisateurs dans les capitules a été mesurée par comptage instantané selon Vaissière et al. (2011): la fréquentation des capitules de tournesol est mesurée grâce à un balayage visuel des capitules au sein de chaque placette. En circulant, l'observateur scanne successivement 100 capitules sur un rang le long de chaque placette, et détermine s'ils sont visités par aucun, un ou plusieurs insectes à ce moment-là. Les insectes sont identifiés et notés selon les catégories suivantes : abeilles domestiques avec ou sans pelotes de pollen, bourdons, autres abeilles sauvages, diptères (syrphes et autres mouches), et autres insectes. La diversité des insectes pollinisateurs a aussi été mesurée sur des transects de $25 \mathrm{~m}$ sur chaque placette (Fig. 2).

Afin de mieux comprendre le comportement des populations d'insectes floricoles, en complément nous avons relevé les données météorologiques et les conditions hydriques du sol car elles peuvent influencer la sécrétion nectarifère des fleurons de tournesol. À chaque date d'observation, les conditions météorologiques (températures moyennes et maximales, l'hygrométrie et la vitesse du vent) étaient enregistrées une fois le matin et une fois l'après-midi. Des prélèvements de nectar ont été effectués du début à la fin de la floraison, sur cinq capitules par parcelle, préalablement mis sous sachet de tulle à la distance moyenne des colonies $(280 \mathrm{~m})$. Ces capitules ont été repérés en début d'anthèse au milieu de la parcelle à proximité des sondes tensiométriques (Fig. 2, capitules orange). Les prélèvements ont été réalisés dans chaque capitule sur huit fleurons au stade mâle. Nous avons ensuite relevé le volume de nectar (en $\mu \mathrm{L})$ et son taux de sucre (en \%) à l'aide d'un réfractomètre adapté (réfractomètre portable Eclipse spécial nectar; Bellingham \& Stanley, Royaume Uni). L'implantation de sondes Monitor R2DX Watermark ${ }^{\circledR}$ permet de mesurer la teneur en eau du sol dans une plage de 10 à 200 centibars avec une résolution de $1 \mathrm{Cb}$. Deux sondes sont 
installées sur chaque parcelle afin de mesurer les tensions hydriques dans deux horizons à 0 à $30 \mathrm{~cm}$ et 30 à $60 \mathrm{~cm}$ de profondeur. Ces mesures étaient enregistrées toutes les 4 heures à partir du stade $E 1$ jusqu'à la récolte sur chaque parcelle. On a aussi mesuré la température du sol à ces profondeurs. Les sondes ont été placées sur la $2^{\mathrm{e}}$ placette à 280 mètres des colonies, à proximité immédiate de là où les prélèvements de nectar sur les capitules ont été réalisés.

Pour compléter les études de pollinisation, trois traitements de pollinisation ont été effectués: pollinisation manuelle saturante (PS), pollinisation sous tulle pour exclure les insectes pollinisateurs avec un biais limité sur l'action du vent (PT) et pollinisation libre (PL). La pollinisation saturante (pollinisation manuelle à l'aide d'un pinceau souple utilisé tous les deux jours avec les fleurs laissées en pollinisation libre) avait pour objectif d'obtenir la charge maximale en pollen et d'exprimer ainsi le potentiel maximum de rendement de la plante dans les conditions de la placette considérée. Ce traitement visait aussi à déterminer si l'on atteignait ce même potentiel en pollinisation libre (autopollinisation passive + pollinisation anémophile + pollinisation entomophile). Le traitement tulle (PT) visait à obtenir uniquement la pollinisation anémophile (par le vent) modifiée

Q6 par la maille du tulle et l'autopollinisation passive. Avec à ce protocole, l'objectif était d'évaluer l'importance de la pollinisation entomophile par rapport aux autres modes de pollinisation. Les capitules correspondant à chaque modalité (PL, PS et PT) ont été marqués par des étiquettes de couleurs distinctes et dans chaque placette, ces traitements ont été effectués en randomisant les traitements dans chaque rang avec cinq capitules par placette et par traitement (Fig. 2).

\section{Q7 4 Mesure de la charge en pollen des stigmates}

Le protocole FAO (Vaissière et al., 2011) a été critiqué parce qu'il ne mesure pas directement le niveau de pollinisation, mais fait l'hypothèse que le niveau de pollinisation est une fonction croissante monotone de l'activité des insectes pollinisateurs (Freitas et al., 2016). Par ailleurs, il est remarquable qu'aucune étude n'ait quantifié l'impact de la pollinisation entomophile sur le niveau de pollinisation du tournesol pour s'affranchir des biais liés aux aléas de la fécondation et du remplissage des akènes (Bos et al., 2007). Pour prendre en compte ces éléments, en 2016 nous avons entrepris de mesurer la charge en pollen des stigmates de tournesol dans cette étude. Nous avons mis au point une méthode pour mesurer la charge en pollen des stigmates de tournesol sur la base de celle mise au point sur les stigmates de melon (Vaissière et Froissart, 1996). Une fois les fleurons fanés, c'est-à-dire au $9^{\mathrm{e}}$ jour après leur anthèse, cinq fleurons sur chaque capitule sont prélevés avec une pince fine et stockés dans des tubes Eppendorf identifiés par des étiquettes. Ces fleurons sont ensuite séchés et disséqués de manière à récupérer leur stigmate. Après un traitement, spécifique les grains de pollen sont colorés et récupérés sur des filtres sur lesquels ils sont dénombrés. La quantité totale de grains de pollen obtenue représente la charge effective en pollen reçu par les stigmates de tournesol au stade femelle à la date du relevé. Cette méthode a été testée sur les stigmates récoltés dix jours Q8 après anthèse une parcelle de relevé.
En 2016, sur une des parcelles, une collecte des stigmates a été réalisée afin d'évaluer la charge en pollen en fonction des distances au front de pollinisateurs et du mode de pollinisation (pollinisation libre, pollinisation sous tulle).

En parallèle des relevés de densité et de diversité d'insectes pollinisateurs, des fleurons au stade femelle sont repérés à l'aide d'épingles à tête colorées de manière à pouvoir ensuite les récolter une fois fanés. Ces suivis sont réalisés sur deux capitules par placette, l'un en pollinisation libre (PL) et l'autre sous tulle (TU).

\subsection{Récolte}

Les capitules avec les différents traitements de pollinisation (PL, PS, PEI) ont été récoltés individuellement et manuellement. De plus, sur chaque placette de relevé dans la zone de récolte nous avons récolté 30 capitules adjacents pour avoir une évaluation du rendement sur chaque placette, soit trois évaluations du rendement par distance.

\section{Résultats}

\subsection{Fréquentation des fleurs}

La fréquentation des fleurons de tournesol a été essentiellement réalisée par les abeilles domestiques, avec très peu de bourdons et d'abeilles sauvages. En 2015, les abeilles domestiques ont représenté environ $85 \%$ des effectifs dans les capitules suivis, les bourdons $11 \%$, les $4 \%$ restant se répartissant entre $2 \%$ d'abeilles sauvages et $2 \%$ de syrphes et autres insectes. Il s'agissait essentiellement d'abeilles domestiques butineuses strictes de nectar (sans pelote de pollen) et ce, quelle que soit la parcelle étudiée

En 2016, nos observations ont renforcé encore cette dominance des abeilles domestiques avec 98,2\% d'abeilles domestiques butineuses de nectar contre $0,06 \%$ d'abeilles domestiques butineuses avec pelotes de pollen, $0,85 \%$ de bourdons, $0,65 \%$ d'autres abeilles sauvages, $0,08 \%$ de syrphes, et $0,13 \%$ d'autres diptères.

\subsection{Gradient d'insectes pollinisateurs}

Aussi bien en 2015 qu'en 2016 il n'a pas été possible d'observer un gradient de pollinisateurs sauvages en raison des faibles populations observées. Seules les activités de butinage des abeilles domestiques à nectar ont pu être étudiées.

En 2015, on a observé un gradient significatif des densités d'abeilles domestiques butineuses de nectar entre $30 \mathrm{~m}$ et $530 \mathrm{~m}$ des colonies. Les densités ont varié de 60 abeilles pour 100 capitules à environ 40 abeilles à $250 \mathrm{~m}$ et un peu plus de 20 abeilles à $530 \mathrm{~m}$ (Fig. 3). L'activité de butinage aussi été réalisée sur des distances intermédiaires (80m 150m). Ces Q9 résultats sont résumés dans la figure 3 chaque couleur de points représente une parcelle d'étude.

En 2016, un gradient n'a pu être observé que sur trois des six parcelles d'étude. Et sur les trois parcelles restantes, on a constaté que le gradient était moins marqué qu'en 2015 (Fig. 4). Et même à $530 \mathrm{~m}$ du front de colonies, on a observé encore une activité conséquente des abeilles domestiques, ce qui suggère que la charge de deux colonies par ha était trop 


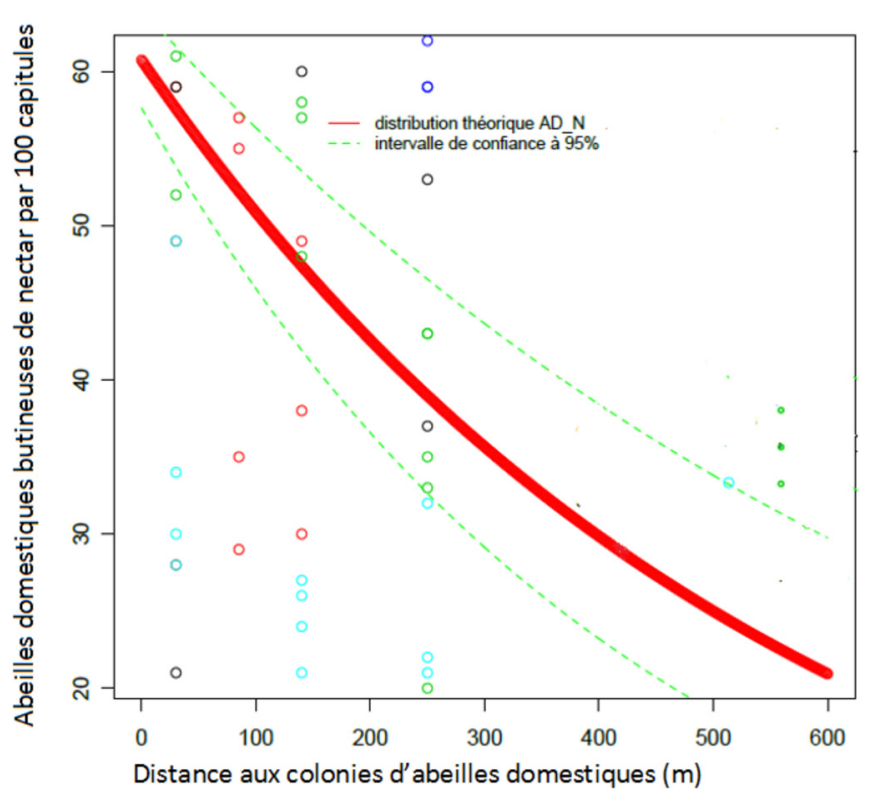

Fig. 3. Gradient des densités d'abeilles domestiques butineuses de nectar sur tournesol en 2015.

Fig. 3. Gradient of density of nectar collecting honey on sunflower in 2015 .

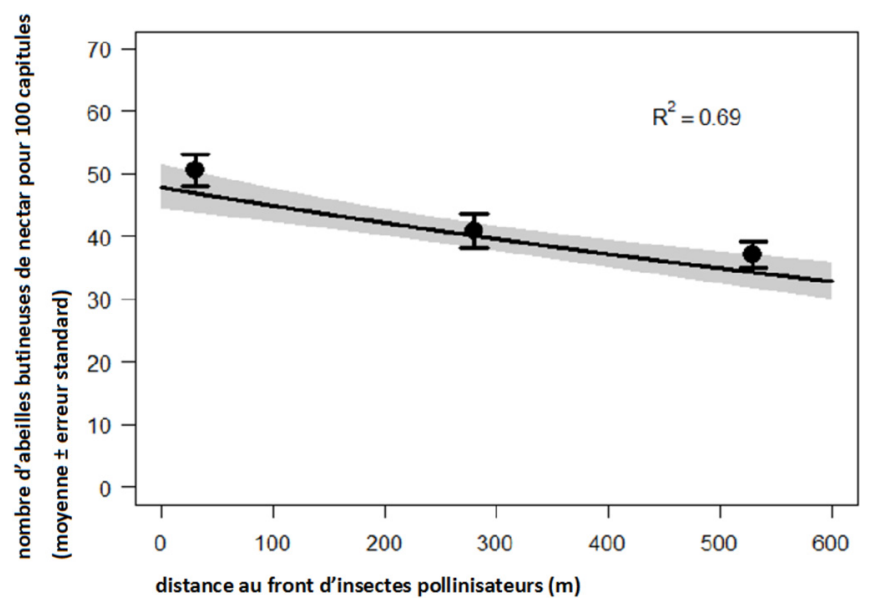

Fig. 4. Gradient d'abeilles domestiques butineuses de nectar en fonction des distances aux colonies dans la parcelle avec le plus de différence en 2016.

Fig. 4. Gradient of density of nectar honeybee foragers based on the distances to the colonies on the field with the most gradient.

élevée pour mesurer un gradient ou qu'il y avait d'autres colonies alentour issues de ruchers que nous n'avons pas détectés dans l'environnement.

\section{La charge en pollen des stigmates}

Il n'y avait pas de gradient significatif de densités de butineuses entre les trois distances du front de colonies à la date choisie pour analyser la charge en pollen des stigmates sur la parcelle d'étude, et, comme attendu, les charges en pollen des stigmates exposées ce jour-là aux trois distances étaient aussi similaires entre elles (moyennes de 340, 334 et 326 grains de pollen/surface réceptive des stigmates exposés à 30,280 et $530 \mathrm{~m}$ des colonies). Il n'y avait pas non plus de différence significative entre les charges en pollen des fleurons exposés aux abeilles (pollinisation libre PL) ou en pollinisation manuelle saturante (PS) indiquant que la densité de 40 abeilles/ 100 capitules observée à $500 \mathrm{~m}$ des colonies le jour d'étude avait permis d'obtenir une pollinisation maximale. Par contre, l'isolation des capitules sous tulle s'est traduite par une réduction très significative de la charge en pollen des stigmates (Fig. 5) démontrant ainsi clairement l'action pollinisatrice des abeilles sur tournesol de consommation.

\subsection{L'effet sur le rendement du tournesol}

En 2015, l'analyse des rendements n'a pu être conduite que sur quatre parcelles parmi les cinq étudiées. Cette dernière ayant été récoltée avant la collecte de nos échantillons. En pollinisation libre, cette analyse a mis en évidence une différence significative entre 30 et $280 \mathrm{~m}$ des colonies (Fig. 6) avec un gain en grains par unité de surface de $17 \%$, un gain de rendement de $30 \%$ et une augmentation de $7 \%$ d'huile. Les capitules de la distance de $530 \mathrm{~m}$ ont été retirés de l'analyse en raison de doute sur l'homogénéité des grains récoltés.

En 2015, les comparaisons des capitules entre les trois traitements de pollinisation (PL, PS, PEI) ont mis en évidence que la pollinisation entomophile était responsable d'un gain de rendement de $42 \%$ en quintaux $/$ ha, de $6,3 \%$ d'huile et de $52 \%$ de graines $/ \mathrm{m}^{2}$ pour la seule variété étudiée (Extrasol). Les différents régimes de pollinisation n'ont pas mis en évidence d'effet sur le PMG probablement en raison d'un effet de compensation lorsque le nombre de grains diminue. La pollinisation saturante (PS) n'a pas entraîné de différence par rapport aux capitules en pollinisation libre (PL). Ceci suggère que l'action des insectes pollinisateurs a permis une pollinisation proche de la saturation.

En 2016, sur les trois parcelles pour lesquelles un faible gradient d'insectes pollinisateurs a été détecté, aucune augmentation significative de rendement attribuable à l'action des pollinisateurs n'a pu être mise en évidence sauf sur une seule parcelle avec un gain de rendement de $12 \%$ entre la distance de $30 \mathrm{~m}$ et celle de $530 \mathrm{~m}$.

\subsection{La nectarification du tournesol}

Certains apiculteurs mettent en cause la sécrétion nectarifère des nouvelles variétés de tournesol, qui serait moins importante que celle des variétés anciennes. En 2015, les études menées n'ont pas permis de juger de l'effet de la génétique, car le suivi de la nectarification n'a été réalisé que sur l'unique variété étudiée (Extrasol). Il s'est avéré toutefois que, pour cette variété, les écarts de nectarification pendant la floraison semblaient principalement liés à l'état hydrique de la couche de sol entre 30 et $60 \mathrm{~cm}$. En cas de stress hydrique, ou de semelle de labour empêchant la descente du pivot de la racine du tournesol dans cet horizon, entraîne une modification de la concentration en sucre du nectar (Fig. 7). Une augmentation de 10 centibars de la 


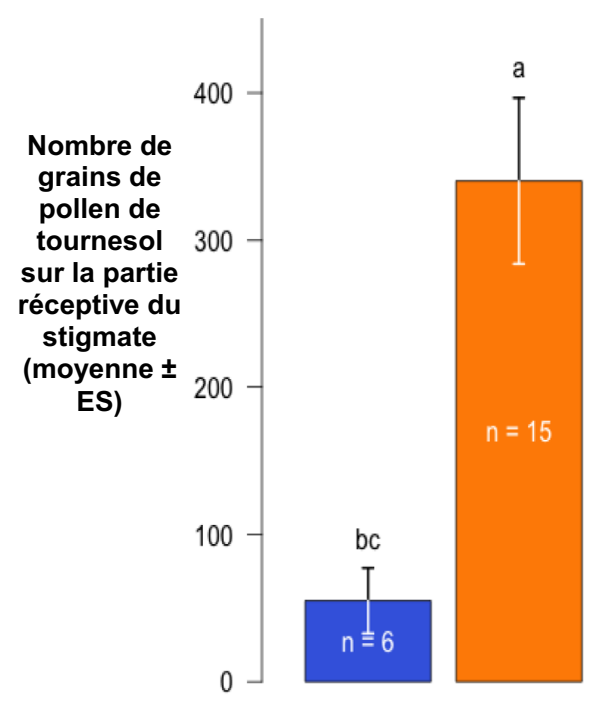

30

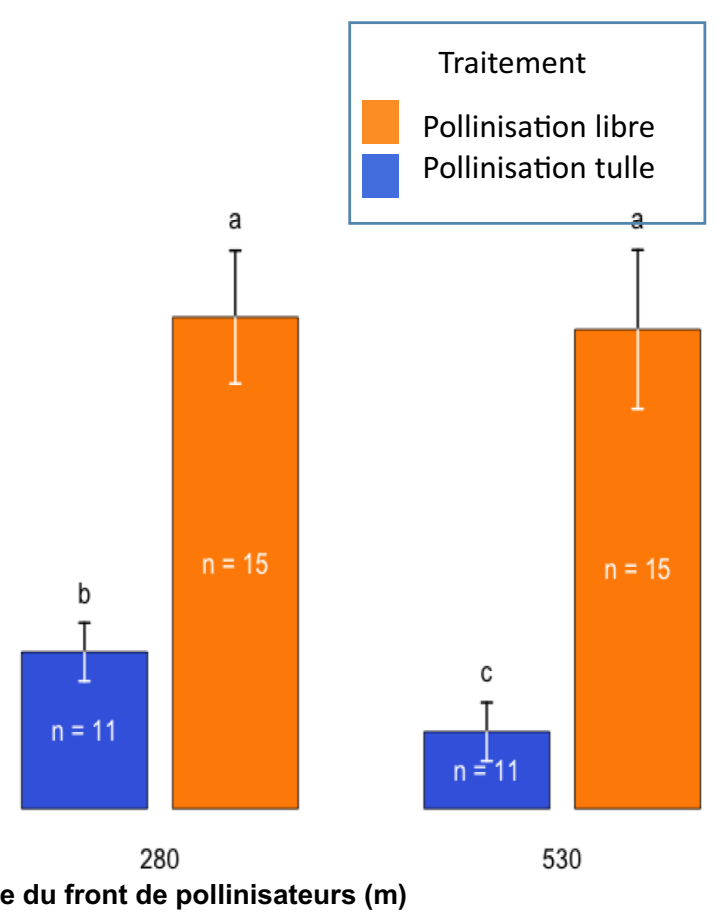

Distance du front de pollinisateurs $(\mathrm{m})$

Fig. 5. Effet de l'activité pollinisatrice des abeilles sur la charge en pollen des stigmates de tournesol de consommation aux trois distances du front de colonies le 26 juillet 2016 sur une parcelle d'étude.

Fig. 5. Effect of the pollinating activity of bees on the stigmatic pollen load of sunflower stigmas at the three distances from the colony front on 26 July 2016 on one study field.

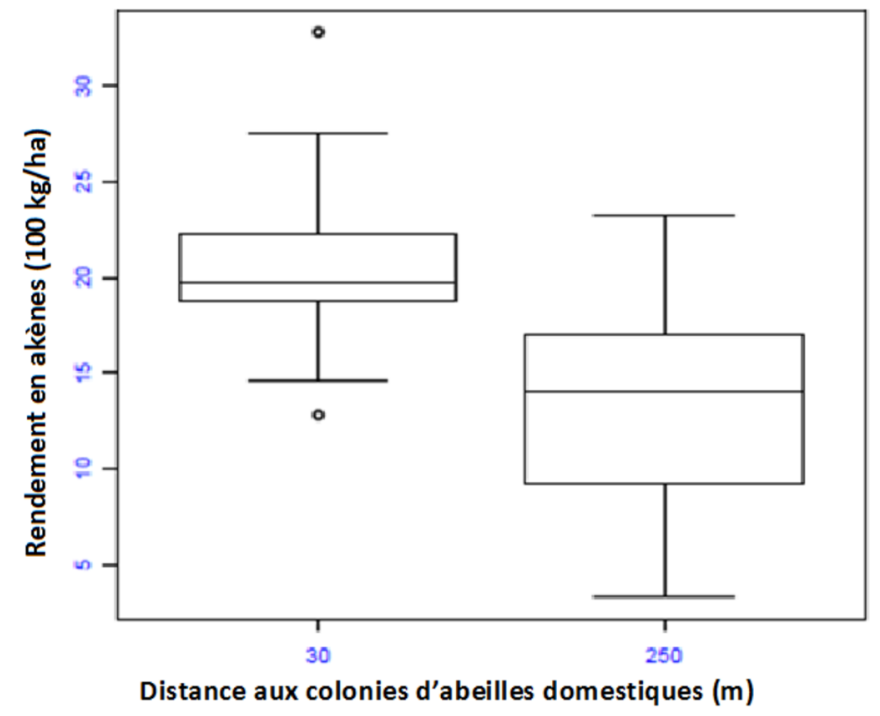

Fig. 6. Box-plot des rendements de tournesol à $30 \mathrm{~m}$ et $280 \mathrm{~m}$ des colonies en 2015. La ligne horizontale indique la moyenne des valeurs à chaque distance.

Fig. 6. Box-plot of the sunflower yields at $30 \mathrm{~m}$ and $280 \mathrm{~m}$ from the colonies of honeybees in 2015. The line indicates the mean value for each distance.

tension hydrique s'est traduite par une diminution de $5 \%$ de la concentration en sucre. En 2015, les parcelles ont été en stress hydriques pendant une grande partie de la floraison du tournesol. Les colonies n'ont pas ou peu produit de miel

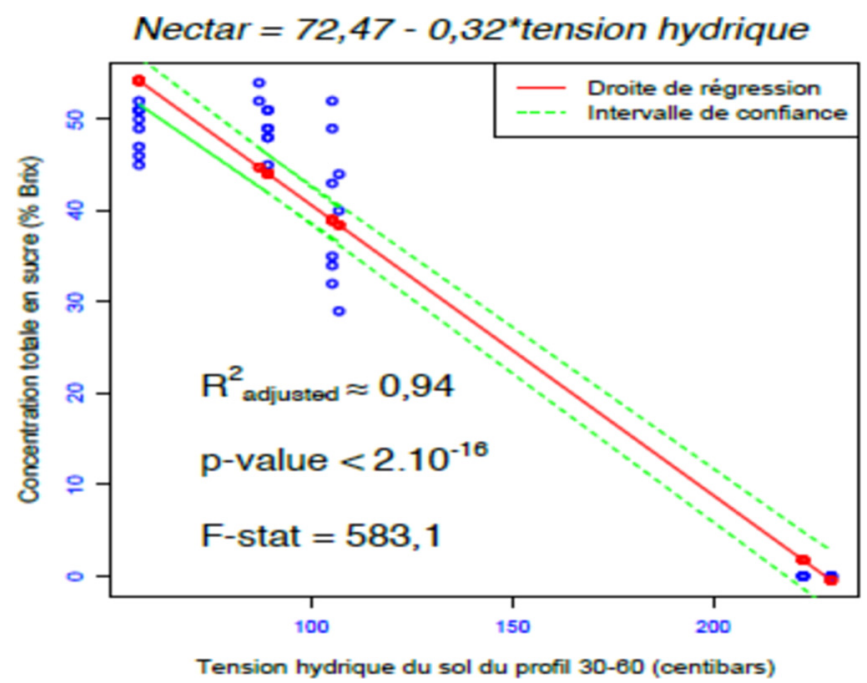

Fig. 7. Relation entre la tension hydrique du sol dans l'horizon 30$60 \mathrm{~cm}$ et la concentration en sucre du nectar (points bleus : valeurs mesurées; points rouges : valeurs du modèle $\mathrm{Y}=72,47-0,32 \mathrm{X} ; \mathrm{r}^{2}$ adjusted $=0,94, P<2.10^{-16}$ ).

Fig. 7. Linear correlation between the water deficit of the soil at 30$60 \mathrm{~cm}$ depth and the sugar concentration of the nectar (blue dots: measured values; red dots: values from the model).

on peut donc s'interroger sur le rôle de l'état hydrique du sol sur la disponibilité du nectar pour les abeilles. Même s'il y a des variations liées à la génétique (Cerrutti et Pontet, 2016), ces stress hydriques qui sont fréquents sur les 
parcelles de tournesol non irriguées pourraient expliquer en partie les arrêts de miellée que des apiculteurs ont constatés.

\section{Conclusions}

Ces études conduites depuis 2015 sur tournesol, se poursuivent en 2017. Elles ont permis de tester et compléter la méthode originale de la FAO (Vaissière et al., 2011) pour mesurer les effets des insectes pollinisateurs en conditions pratiques agricoles.

Avec des charges de deux colonies d'abeilles domestiques par hectare, la fréquentation des capitules de tournesol est largement dominée par les abeilles domestiques (plus de $85 \%$ ). Et nos études ont permis de montrer que ces insectes assurent une bonne pollinisation du tournesol de consommation même à la densité de 40 abeilles domestiques/100 capitules. La nectarification s'est avérée très dépendante des conditions hydriques pendant la floraison, notamment dans l'horizon $30-60 \mathrm{~cm}$ ou de l'accès du pivot des plantes à cet horizon.

Un gain de rendement en fonction de la distance aux colonies d'abeilles domestique a été observé entre $30 \mathrm{~m}$ et $280 \mathrm{~m}$

Par ailleurs, un gain de rendement de $40 \%$ a été mesuré en faveur de la pollinisation entomophile, accompagné d'un gain en huile de six points comparé aux capitules sous tulle (autopollinisation passive plus vent) en 2015 sur une variété. Cette étude, poursuivie en 2016, n'a pas confirmé de gain de rendement significatif sur cinq des six parcelles d'étude du fait de l'abondance des densités d'abeilles sur l'ensemble des distances et de l'aptitude des variétés à l'autofertilité. Une seule parcelle a exprimé un gain de rendement modeste de $12 \%$. Toutefois, suite à ces deux années d'études, on constate qu'avec une charge limitée à deux colonies par hectare les abeilles sont observées à $530 \mathrm{~m}$ et que donc cette distance ne peut pas être strictement considérée comme exempte de pollinisation entomophile. La poursuite de ces études en 2017 devrait permettre d'affiner ces premières tendances

\section{Références}

Barbier E, Abid M. 1966. Pollinisation et formation des graines chez le tournesol (Hélianthus annuus L.). Al Awamia 21: 63-83.

Bos MB, Veddeler D, Bogdanski AK, et al. 2007. Caveats to quantifying ecosystem services: fruit abortion blurs benefits from crop pollination. Ecol Applic 17: 1841-1849.

Carvalheiro LG, Veldtman R, Shenkute AG, et al. 2011. Natural and within-farmland biodiversity enhances crop productivity. Ecol Lett 14: 251-259.

Cerrutti N, Pontet C. 2016. Differential attractiveness of sunflower cultivars to the honeybee Apis mellifera L. OCL 23(2): D204. Available from https://www.ocl-journal.org/articles/ocl/pdf/ 2016/02/ocl160005-s.pdf.

Freitas BM, Vaissière BE, Saraiva AM, Carvalheiro LG, Garibaldi LA, Ngo H. 2016. Identyfying and assessing pollination deficits in crops. In: Gemmill-Herren B, ed. Pollination services to agriculture. New York: FAO, Routledge, pp. 17-33.

Garibaldi LA, Steffan-Dewenter I, Winfree R, et al. 2013. Wild pollinators enhance fruit set of crops regardless of honeybee abundance. Science 339: 1608-1611.

GNIS. 2016 fiche filière semences de tournesol [en ligne].

Krishna V, Prashanth Y, Yojeeswarudu B, Maurya K. 2014. Pollination efficiency of honeybees in sunflower (Helianthus annuus L). J Agric Life Sci 1(2): 92-95.

Leonhardt SD, Gallai N, Garibaldi LA, Kuhlmann, Klein A-M. 2013. Economic gain, stability of pollination and bee diversity decrease from southern to northern Europe. Basic Appl Ecol 14: 461-471.

Nderitu J, Nyamasyo G, Kasina M, Oronje ML. 2008. Diversity of sunflower pollinators and their effect on seed yield in Makueni District, Eastern Kenya. Spanish J Agricul Res 6: 271-278.

ONIDOL. 2012 et 2013. Interprofession des oléagineux, chiffres clés, Oléagineux France, 2012-2013.

Taséi JN. 1984. Cultures à graines oléagineuses des régions tempérées. In : Pesson P, Louveaux J, eds. Pollinisation et productions végétales. Paris : INRA, pp. 309-330.

Vaissière BE, Froissart R. 1996. Pollination of cantaloupes under spunbonded row cover by honeybees (Hymenoptera: Apidae) in West Africa. J Econ Entomol 89: 1213-1222.

Vaissière B, Freitas B, Gemmill-Heren B. 2011. Protocol to detect and assess pollination deficits in crops: a handbook for its use. Rome: F.A.O, p. 70 .

Citation de l'article : Fougeroux A, Leylavergne S, Guillemard V, Geist O, Gary P, Cenier C, Caumes-Sudre E, Senechal C, Vaissière B. 2017. Effet de l'activité des insectes pollinisateurs sur la pollinisation et le rendement du tournesol de consommation. OCL 24(6): D603. 